\title{
Contribución de los datos genómicos en la definición de la composición racial de bovinos doble propósito
}

\author{
Jaime Anibal Rosero Alpala ${ }^{a^{*}}$ \\ Wilson David Rangel Garcia ${ }^{\text {a }}$ \\ Adonai Rojas Barreto ${ }^{a}$ \\ William Orlando Burgos-Paz ${ }^{\mathrm{b}}$
}

${ }^{a}$ Corporación Colombiana de Investigación Agropecuaria-AGROSAVIA, Centro de Investigación La Libertad, km 17 vía Puerto López, Villavicencio, Meta, Colombia.

${ }^{\mathrm{b}}$ Corporación Colombiana de Investigación Agropecuaria - AGROSAVIA, Centro de Investigación Tibaitatá, Mosquera, Cundinamarca, Colombia.

*Autor de correspondencia: jroseroa@agrosavia.co

\section{Resumen:}

La heterosis animal es clave en la obtención de animales más productivos y mejor adaptados al trópico. Sin embargo, el inadecuado manejo genético conlleva a la obtención de mosaicos de razas que incluye la pérdida del potencial productivo del hato. El objetivo de este estudio fue definir la composición racial de bovinos mestizos doble propósito en el piedemonte Llanero departamento del Meta-Colombia. Un total de 126 individuos mestizos (MEZ) de seis hatos fueron evaluados por una aproximación fenotípica (APF) y una genotípica (ARG). Para ARG se incluyeron las razas control asociadas a Bos taurus taurus y Bos taurus indicus, para ello se genotiparon con un chip de GeneSeek GGP-LD de $26 \mathrm{~K}$ de SNP y se analizaron mediante análisis de componentes principales (PCA) y asignación probabilística bayesiana de ADMIXTURE. Las agrupaciones raciales generadas por APF variaron respecto de ARG. El análisis molecular detectó siete $(\mathrm{k}=7)$ grupos genéticos en la composición racial de los animales de estudio. Las tres razas con mayor participación en la composición racial de los individuos mestizos fueron: Holstein, Gyr, Brahman con 23.4, 21.4, y $21 \%$ respectivamente, mientras que las restantes Blanco Orejinegro, Pardo Suizo, Normando y Jersey no superaron el $13 \%$. A diferencia de APF, la aproximación ARG permitió de forma efectiva la identificación de la composición 
racial de bovinos mestizos y suministró información clave en miras de desarrollo de programas de apareamiento que buscan mejorar indicadores productivos, y a su vez propendan por la adaptación de los animales requisito indispensable para los sistemas bovino doble propósito.

Palabras clave: Genotipado, Mestizos, Variabilidad, Genética.

Recibido: 13/05/2020

Aceptado: 29/03/2021

\section{Introducción}

El sistema bovino doble propósito en Colombia representa aproximadamente el $35 \%$ (8.2 millones de cabezas) de la población bovina total ${ }^{(1)}$. Similar a otras regiones de América latina, este sistema se basa en la obtención de animales a partir de cruzamiento de razas con algún grado de ventaja productiva en un entorno particular, y que le confiera mayor $\operatorname{productividad}^{(2)}$.

Contrario a lo obtenido en condiciones subtropicales, los sistemas de producción bovina en general en regiones tropicales no han alcanzado el éxito esperado ${ }^{(3)}$ y como recurso para mejorar la producción se realiza la selección de razas locales y exóticas de los

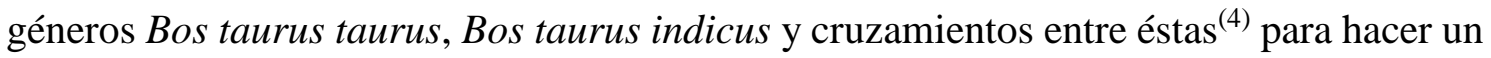
uso eficiente de la heterosis, paterna o materna según se utilice, e incrementar la eficiencia de los sistemas de producción de carne o leche ${ }^{(5)}$.

Sin embargo, no todos los cruzamientos pueden conferir las ventajas esperadas y la incorrecta aplicación de lineamientos zootécnicos puede acelerar la presencia de problemas de adaptación y producción. Es necesario considerar las implicaciones de un hato multirracial, donde su composición racial es parcialmente conocida o desconocida completamente, y donde los efectos genéticos no aditivos delinean la expresión del fenotipo animal ${ }^{(6)}$.

$\mathrm{Al}$ respecto, la situación en Colombia requiere una atención especial, pues además de una condición tropical predominante, el uso de una amplia gama de razas y cruces, la falta de registros productivos y el uso indiscriminado de reproductores sin conocimiento de la procedencia o el manejo, resultan en una errónea percepción de animales más productivos y mejor adaptados a las condiciones donde se explotan ${ }^{(4,6)}$.

En este caso, la disponibilidad de información de la estructura genealógica o co-ancestría entre individuos, permite manejar la diversidad y a tener un control sobre la endogamia ${ }^{(7)}$ 
y el uso de marcadores moleculares, particularmente de SNP (del inglés Single Nucleotide Polymorphism), ha demostrado la efectividad de los análisis genómicos en la determinación de la composición racial en hatos mestizos de carne $^{(8)}$ y leche ${ }^{(9)}$ con deficiente información genealógica.

De hecho, la contribución de los análisis moleculares ha permitido identificar si el origen B. taurus taurus o B. taurus indicus de un segmento cromosómico de bovinos puede tener efectos en características de interés productivo ${ }^{(10)}$.

Por lo anterior, el objetivo de este estudio fue cuantificar la contribución de la información genómica, en la determinación de la composición racial de bovinos mestizos del sistema doble propósito predominante en el piedemonte Llanero, como herramienta de apoyo en la definición de estrategias de manejo y selección en hatos mestizos de la región.

\section{Material y métodos}

\section{Localización}

Este estudio se realizó en animales presentes en seis hatos de tres rutas lecheras en la subregión del piedemonte Llanero del departamento del Meta- Colombia. Esta subregión se caracteriza por presentar temperaturas que oscilan entre 23 y $30{ }^{\circ} \mathrm{C}$, humedad relativa entre 76 y $78 \%$ y alturas a nivel del mar entre 300 y $700 \mathrm{msnm}^{(11)}$.

Los hatos mestizos (MEZ) en cada municipio definidos se nombraron por siglas de la siguiente manera: ACA: hato municipio de Acacías, CLN: hato municipio de Castilla La Nueva, CUM: hato municipio de Cumaral, MES: hato municipio de Mesetas, SJA: hato municipio de San Juan de Arama y VLL: hato municipio de Villavicencio.

\section{Valoración fenotípica}

Inicialmente, en cada hato seleccionado (conformados por un promedio de 70 animales), se consolidó la información productiva mediante una encuesta básica para identificar los criterios productivos y objetivos de uso de las razas presentes. Posteriormente, se llevó a cabo la clasificación racial de un grupo de animales representativos y del cual hacían parte novillas y vacas de hasta tercer parto. Para tal fin, se seleccionaron alrededor de 21 animales por hato, para un total de 126 animales con el ánimo de generar una clasificación por su Aparente predominancia fenotípica $-\mathrm{APF}^{(12)}$, donde se tuvo en cuenta la amplia gama de cruzamientos entre razas de origen B. taurus taurus y B. taurus indicus usadas en los hatos del sistema de producción doble propósito. La APF clasifica los animales así: animales con predominio B. taurus taurus (PREDTAU): sin giba, sin papada y sin pliegue 
umbilical, orejas cortas y peludas, pelaje manchado o no, pelaje negro, rojo y marrón, con cuernos o sin ellos. Animales con predominio B. taurus indicus (PREDCEB): con giba, papada y pliegue umbilical muy desarrollado, orejas largas y sin pelos, color sólido, gris, negro, cenizo o rojo. Con predominancia intermedia $B$. taurus taurus $\mathrm{x}$ B. taurus indicus (PREDINTER): con giba, papada y pliegue umbilical, orejas largas y escasos pelos, raramente manchado, casi siempre con cuernos.

\section{Asignación racial genotípica}

La asignación racial genotípica-ARG, se obtuvo a partir información genotípica de 126 animales (21 por hato), previamente seleccionados (por APF) y muestreados bajo las recomendaciones de un muestreo reducido extremo ${ }^{(13)}$, así garantizar la comparación entre las dos matrices de información (APF y ARG). Para la valoración molecular, en cada animal se colectó una muestra de sangre mediante punción en la vena coccígea y se transportaron al Laboratorio de Genética Molecular del Centro de Investigación Tibaitatá de AGROSAVIA para su posterior genotipificación. La extracción de ADN se realizó mediante el kit comercial UltraClean ${ }^{\circledR}$ Blood DNA Isolation (MoBio Laboratories Inc.) y el genotipado de polimorfismos de nucleótido simple (SNP) distribuidos en el genoma bovino se realizó con el chip GGP-LD de GeneSeek® de 26K SNPs bajo las recomendaciones de la casa fabricante.

\section{Depuración de bases de datos y análisis estadístico}

La información derivada de la evaluación fenotípica permitió generar cuadros de frecuencia sobre los componentes raciales observados en los hatos evaluados. Por su parte, la información molecular fue preparada para análisis poblacionales, a fin de identificar los grupos genéticos presentes. Por tanto, inicialmente se excluyeron aquellos marcadores SNP con posición desconocida y los ubicados en cromosomas sexuales. Igualmente se descartaron del análisis SNP que no fueron detectados en más de $5 \%$ de los individuos, aquellos SNP que se desviaron del equilibrio Hardy-Weinberg $(P<0.01)$ y los SNP que presentaron un MAF <0.01. Después de realizar la depuración se emplearon 24,266 SNP de las 126 muestras, con los que se realizaron los análisis genéticos.

Con la información molecular se determinó inicialmente la estructura genética de la población MEZ mediante el análisis de componentes principales (PCA), es decir, a partir de los datos genotípicos se evaluó las relaciones genéticas entre individuos y se buscó posibles aglomeraciones respecto a los grupos genéticos establecidos en APF y las razas control usadas en ARG. Para tal fin, se usó el comando PRCOMP de la librería STATS del programa $\mathrm{R}^{(14)}$. Para identificar las relaciones genéticas y posibles eventos de introgresión se utilizó la asignación probabilística de individuos a grupos genéticos, para ello, se empleó el algoritmo de máxima verosimilitud, el cual, basado en modelos estima 
la ancestria y calcula la probabilidad de los genotipos observados usando las proporciones de ancestria y las frecuencias alélicas de la población, algoritmo implementado en ADMIXTURE $^{(15)}$. Para la determinación de los $\mathrm{k}$ grupos genéticos presentes en la población, se analizaron de $\mathrm{k}=2 \mathrm{a} \mathrm{k}=10$ grupos genéticos, y el valor k más probable se determinó por el valor más bajo de error en la validación cruzada realizada con las opciones por defecto del programa, y las recomendaciones del autor ${ }^{(15)}$.

Finalmente, mediante el algoritmo descrito previamente ${ }^{(15)}$, se compararon las frecuencias alélicas de los SNP de la población mestiza de este estudio (MEZ, n= 126), respecto a las razas control, donde se usó un número aproximado de 20, buscando simetría con el número de muestras tomadas por hato (21 muestras). Para ello, se usó una base de datos de SNPs de las siete razas control, facilitadas por Laboratorio de Genética Molecular del Centro de Investigación Tibaitatá de AGROSAVIA y correspondieron a las razas: Brahman (BRA, $n=18)$, Holstein (HOL, $n=29)$, Blanco Orejinegro (BON, n= 19), Gyr (GYR, n= 18), Pardo Suizo (PAR, $n=20)$, Jersey (JER, $n=20$ ) y Normando NOR, $n=20$ ) por considerar su aparente uso en la formación del mestizaje de los bovinos del sistema doble propósito y de este modo establecer los grupos genéticos con mayor precisión.

\section{Resultados}

\section{Valoración fenotípica}

A partir de la información suministrada por los productores, se encontró que los grupos raciales predominantes fueron cruces con Gyr (22.4 \%), cruces con Cebú (18.36 \%), cruces con Holstein (16.32 \%), cruces con Pardo Suizo (10.20 \%), cruces con Normando $(4.76 \%)$ y cruces con Jersey $(4.08 \%)$. En la mayoría de los casos, los productores respondieron que la aparente composición racial de sus hatos obedecía a la raza del toro reproductor usado en los últimos años de apareamientos. El restante $23.80 \%$ de los cruces hacen referencia a individuos con posibles grupos raciales criollos y no definidos.

Por su parte, en los hatos MEZ con la aproximación APF, el $34 \%$ de los animales fueron catalogados como PREDCEB, $37 \%$ como PREDTAU y el restante $29 \%$ como PREDINTER. A nivel de los hatos, las proporciones de las agrupaciones por fenotipo fueron variables entre sí, aunque una ligera similitud fue observada entre SJA y CUM (Figura 1), que a pesar de estar en regiones distantes muestran composiciones genéticas similares debido a su manejo y orientación productiva. 
Figura 1: Porcentaje de contribución por agrupaciones fenotípicas en los hatos evaluados

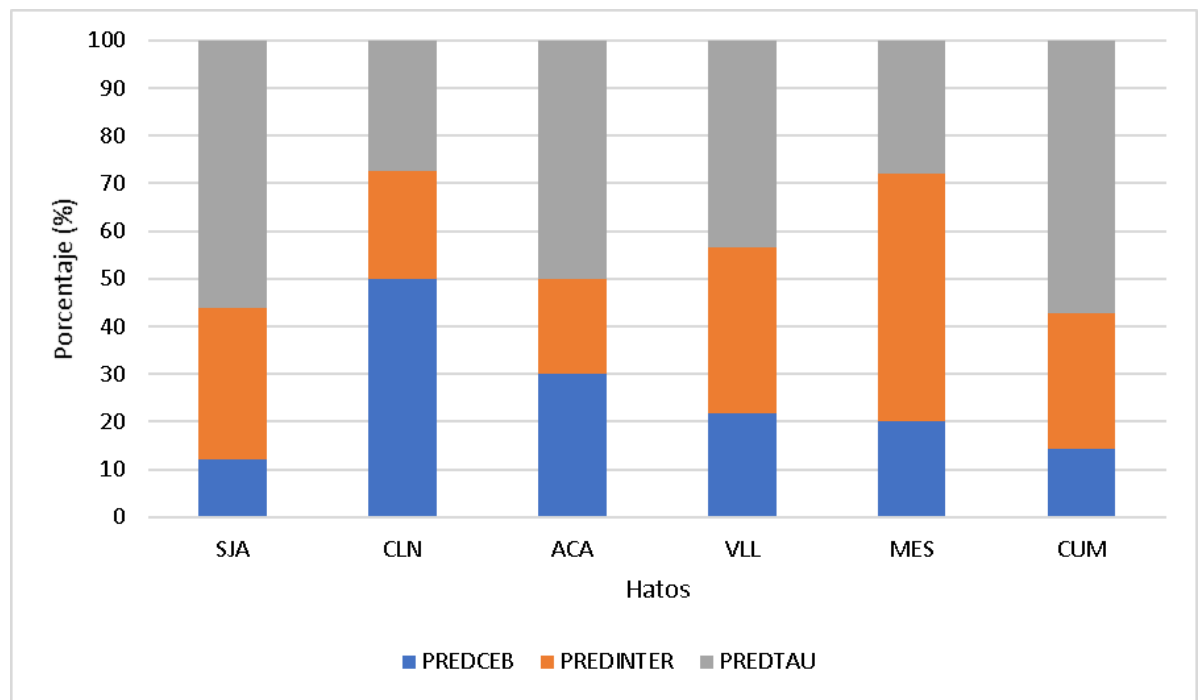

PREDCEB = animales con predominio Bos taurus indicus, PREDTAU $=$ animales con predominio Bos taurus taurus, PREDINTER= animales con predominancia intermedia Bos taurus taurus $\mathrm{x}$ Bos taurus indicus, $\mathrm{ACA}=$ hato municipio de Acacías, $\mathrm{CLN}=$ hato municipio de Castilla La Nueva, CUM= hato municipio de Cumaral, MES= hato municipio de Mesetas, SJA= hato municipio de San Juan de Arama y $\mathrm{VLL}=$ hato municipio de Villavicencio.

\section{Asignación racial genotípica}

En primer lugar, con la información genotípica se estableció la relación genética entre las muestras mediante análisis de componentes principales para los tres grupos genéticos PREDTAU, PREDINTER y PREDCEB (Figura 2). El primer componente principal (PC1) explicó el $6.52 \%$ de la varianza total, asociada a la diferenciación de los componentes genéticos Bos taurus taurus y Bos taurus indicus. Las agrupaciones por APF propuestas PREDTAU, PREDCEB y PREDINTER no mostraron la separación genética esperada para la población MEZ, y por el contrario, todos los individuos de los grupos se dispersaron a lo largo del primer componente. Por su parte, el segundo componente principal explicó el $2.9 \%$ de la variación, donde un grupo de individuos valorados por APF como PREDTAU se separó del eje de diferenciación observado en PC1 (Figura 2). En las gráficas de PCA se observó una alta dispersión en los animales con fenotipo Bos taurus taurus y Bos taurus indicus, lo cual puede asociarse a una variabilidad genética de las poblaciones superior a la variación fenotípica. No obstante, la proyección espacial de los animales diferenciados por el PC2 es un indicador de cómo las frecuencias alélicas distinguen animales con predominancia de un fenotipo, lo que hace obligatorio el uso de razas control para su mejor definición. 
Figura 2: Análisis de componentes principales (PCA) entre individuos para bovinos mestizos doble propósito y su aparente predominancia fenotípica (APF)

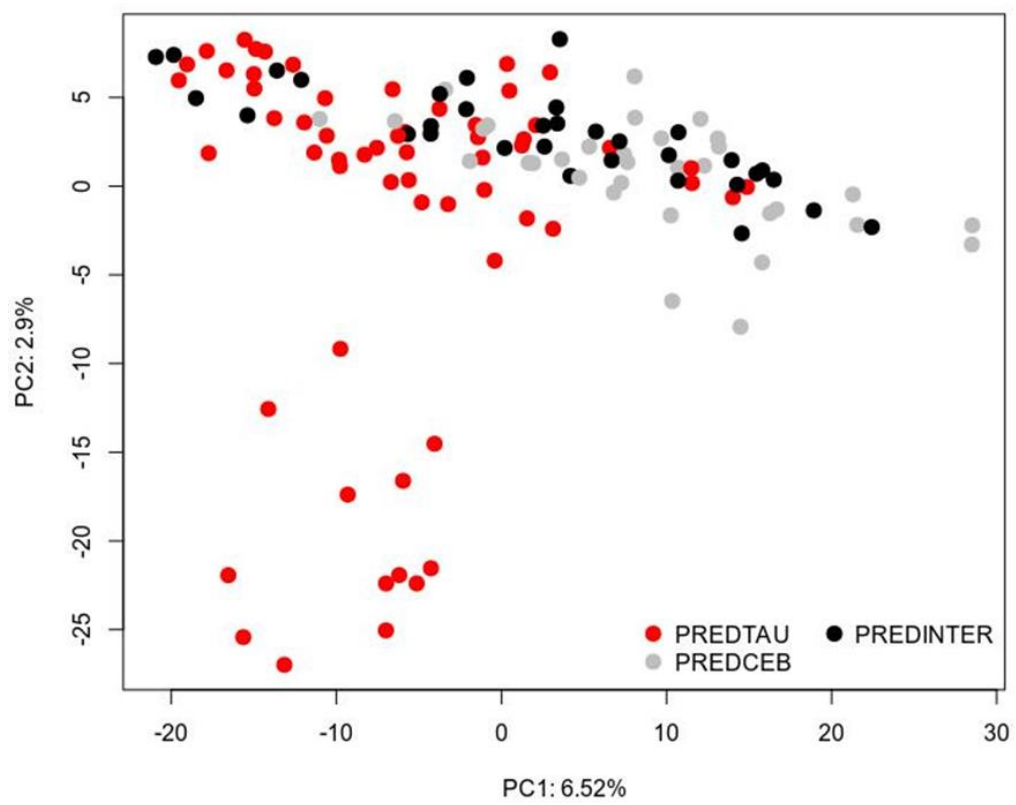

PREDCEB = animales con predominio Bos taurus indicus, PREDTAU $=$ animales con predominio Bos taurus taurus, PREDINTER= animales con predominancia intermedia Bos taurus taurus $\mathrm{x}$ Bos taurus indicus.

Con el fin de establecer posibles razas que conformen el grupo genético el grupo MEZ, se incluyeron siete razas control y se valoró su relación mediante PCA (Figura 3).

Figura 3: Análisis de componentes principales (PCA) con la inclusión de grupos raciales testigo en la población de bovinos doble propósito mestizo (MEZ)

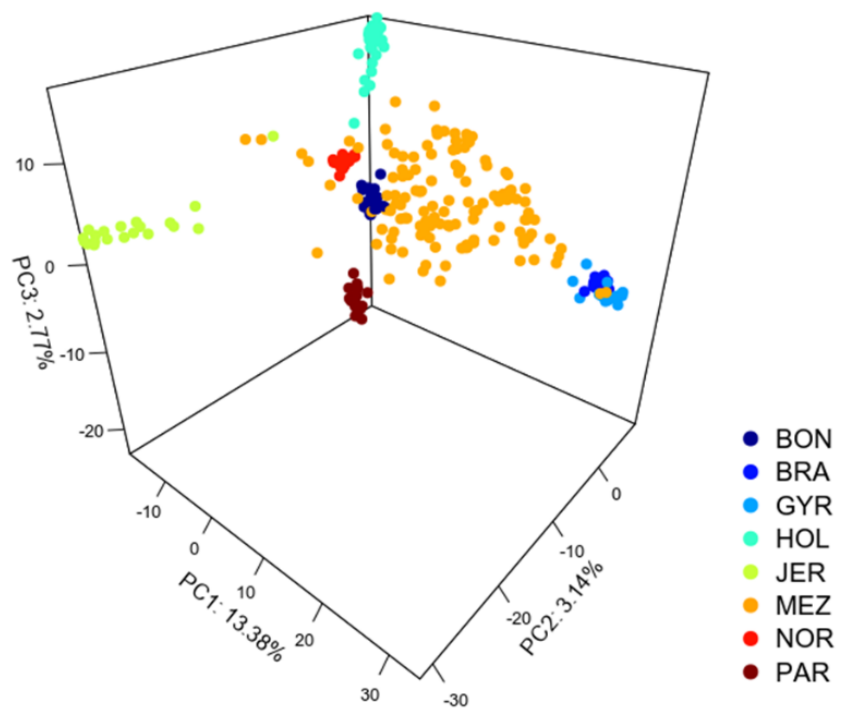

$\mathrm{BON}=$ Blanco Orejinegro, BRA= Brahman, GYR= Gyr, HOL $=$ Holstein, JER $=$ Jersey, NOR= Normando, PAR= Pardo Suizo. 
El PC1 explicó 13.38 \% de la variación total asociada a la diferenciación entre animales del grupo de razas B. taurus indicus (BRA y GYR) ubicados al lado derecho y el grupo de razas $B$. taurus taurus (HOL, JER, PAR, BON y NOR) ubicado al lado izquierdo (Figura 3). De hecho, la proyección espacial de los individuos mestizos presenta un amplio espectro entre estos dos grupos de razas tal como se observó inicialmente en la APF.

El segundo componente principal PC2 explicó el 3.14 \% de la variación. Es evidente que el grupo de razas $B$. taurus taurus presenta mayor variabilidad que el grupo $B$. taurus indicus, se destaca que la raza JER muestra la mayor separación entre las razas $B$. taurus taurus y solo un pequeño grupo de animales MEZ se mostrarían en el espectro hacia esta raza (Figura 3). El tercer componente-PC3, el 2,77 \% de la variación fue explicada por este componente, las razas HOL y PAR se muestran como los grupos más alejados entre sí y en el espectro abarca a una parte de los animales mestizos de este estudio y a animales de las razas BON y NOR.

La segunda aproximación para definir los grupos genéticos existentes empleó la asignación probabilística bayesiana implementada en ADMIXTURE, donde se determinó que el menor error en la validación cruzada correspondía a $7(\mathrm{k}=7)$ grupos genéticos, tomando este valor como adecuado para explicar la composición genética en la población MEZ de este estudio, asociada a los grupos genéticos BRA, GYR, BON, HOL, PAR, JER y NOR (Figura 4), donde se observa la representación gráfica de los resultados, donde cada individuo está representado por una línea vertical, y los colores representan la fracción de asignación a cada grupo genético.

Figura 4: Estructura poblacional de genotipos de la población mestiza doble propósito (MEZ) y razas control

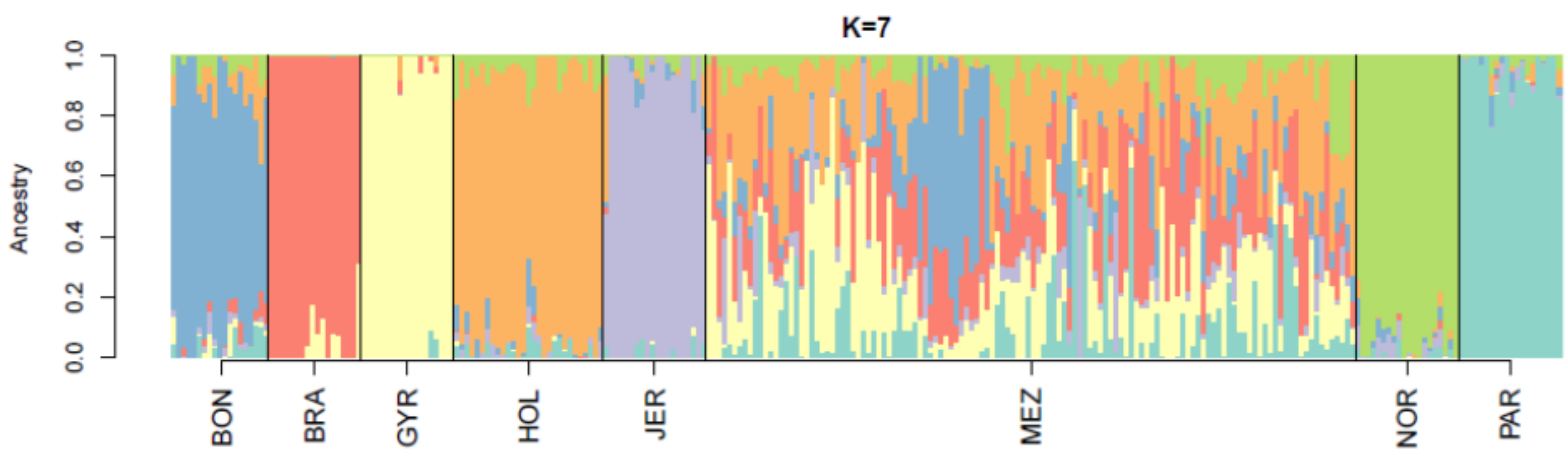

$\mathrm{BON}=$ Blanco Orejinegro, BRA $=$ Brahman, GYR= Gyr, HOL= Holstein, $\mathrm{JER}=$ Jersey, NOR= Normando, PAR= Pardo Suizo.

Las razas HOL, GYR y BRA presentaron la mayor proporción racial en la población MEZ. El análisis de ADMIXTURE permitió con mayor claridad determinar la composición racial del hato de estudio respecto a los análisis de PCA. Sin embargo, ciertos hatos mostraron de forma excepcional composiciones raciales abundantes para ciertas razas como la $\mathrm{BON}$. 
En el Cuadro 1, se observa la composición racial por hato a partir de la información generada del análisis de ADMIXTURE. Se encontró que la contribución de las razas HOL, GYR y BRA en la composición de MEZ fue $23.47 \%, 21.43 \%$ y $21.05 \%$ respectivamente. Otras razas como BON (12.41\%) y PAR (10,15\%) contribuyeron en menor medida, y las razas NOR y JER mostraron la menor contribución de todas las razas testigo con 6.51 y $4.96 \%$ respectivamente. Las razas HOL, GYR y BRA fueron predominantes en las observaciones fenotípica y molecular de la población MEZ, pero la estimación de su aporte al acervo genético de la población mestiza mejoró considerablemente cuando se usaron los análisis moleculares.

Cuadro 1: Porcentajes de conformación racial de bovinos mestizo doble propósito (MEZ) respecto a razas control

\begin{tabular}{lrrrrrrr}
\hline Hato* & PAR & GYR & JER & BRA & BON & HOL & \multicolumn{1}{c}{ NOR } \\
\hline ACA & 10.99 & 23.41 & 2.84 & 16.65 & 6.33 & 32.18 & 7.59 \\
CLN & 9.70 & 36.07 & 4.08 & 14.39 & 3.58 & 28.05 & 4.13 \\
CUM & 19.12 & 15.52 & 10.04 & 18.96 & 8.54 & 22.38 & 5.42 \\
MES & 12.93 & 18.14 & 3.36 & 28.92 & 5.62 & 25.05 & 5.98 \\
SJA & 7.03 & 22.58 & 5.94 & 18.12 & 6.29 & 28.02 & 12.01 \\
VLL & 3.04 & 14.58 & 2.87 & 25.76 & 41.07 & 9.41 & 3.29 \\
Total general & 10.15 & 21.43 & 4.96 & 21.05 & 12.41 & 23.47 & 6.51 \\
\hline \multicolumn{7}{c}{ PAR= Pardo Suizo, GYR= Gyr, JER= Jesey; BRA= Brahman, BON= Blanco Orejinegro, HOL= } \\
*Hato: ACA= hato mestizo municipio de Acacías, CLN= hato mestizo municipio de Castilla La Nueva, \\
CUM= hato mestizo municipio de Cumaral, MES= hato mestizo municipio de Mesetas, SJA= hato \\
mestizo municipio de San Juan de Arama, VLL= hato mestizo municipio de Villavicencio.
\end{tabular}

De igual forma el uso de marcadores moleculares permitió cuantificar la proporción de otras razas cuyo aporte por fenotipo resultan menos predecibles. Por ejemplo, se identificó la alta presencia de la raza criolla colombiana BON en VLL (41.07\%); presencia de la raza PAR en CUM (19.12 \%), MES (12.93\%) y ACA (10.99\%), mientras que NOR sobresalió en SJA (12.01\%) y JER en CUM (10.04 \%) como se observa en el Cuadro 1.

\section{Aparente predominancia fenotípica (APF) vs Asignación racial genotípica (ARG)}

Cuando los tres grupos genéticos generados por la aparente predominancia fenotípicaAPF (PREDTAU, PREDCEB y PREDINTER) fueron comparados con las composiciones raciales generadas por la asignación racial genotípica-ARG, se encontró que los animales asignados al grupo PREDTAU (37\% de la población de estudio), presentaban una amplia variación de su composición racial obtenida mediante ARG. Los grupos raciales usados como testigos en ARG, en las razas variaron entre un mínimo de $0.1 \%$ hasta un máximo de $80 \%$, el valor más alto de asignación fue encontrado en la 
raza $\mathrm{BON}$, seguido de PAR y $\mathrm{HOL}$, no obstante, la raza GYR también mostró una considerable proporción (Cuadro 2).

Cuadro 2: Asignación racial genotípica (ARG) individual máxima, respecto a los grupos raciales asignados por aparente predominancia fenotípica (\%)

\begin{tabular}{llllllll}
\hline Grupos APF $^{*}$ & PAR & GYR & JER & BRA & BON & HOL & NOR \\
\hline PREDCEB & 35.78 & 84.27 & 34.57 & 71.66 & 18.34 & 53.34 & 14.26 \\
PREDINTER & 44.42 & 60.41 & 15.39 & 58.34 & 88.07 & 56.09 & 34.84 \\
PREDTAU & 65.16 & 65.40 & 53.46 & 52.73 & 80.01 & 64.86 & 33.49 \\
\hline
\end{tabular}

BON= Blanco Orejinegro, GYR= Gyr, NOR= Normando, BRA= Brahman, JER= Jersey, HOL= Holstein, $\mathrm{PAR}=$ Pardo Suizo.

*APF $=$ aparente predominancia fenotípica, $\mathrm{PREDCEB}=$ con predominio Bos taurus indicus, PREDTAU= con predominio Bos taurus taurus, PREDINTER=con predominancia intermedia Bos taurus taurus $x$ Bos taurus indicus.

Por su parte, animales catalogados en PREDCEB (34 \% de la población de estudio), mostraron una variabilidad en la composición racial principalmente para las razas relacionadas al grupo Bos taurus indicus, con valores que iban desde un mínimo de $0.1 \%$ hasta un máximo de $84.2 \%$, se destacó las máximas asignaciones en la raza GYR y BRA, e incluso se desataca la considerable asignación encontrada para HOL (Cuadro 2).

Para animales agrupados en PREDINTER (29\% de la población de estudio), cuya asignación racial predominante presenta mayor dificultad, mostraron una gran variabilidad tanto para las razas Bos taurus indicus como para las razas Bos taurus taurus de acuerdo con ARG. Los valores de asignación racial variaron entre 0.1 y $88 \%$, para razas BON y HOL y valores relevantes de asignación en las razas GYR y BRA (Cuadro 2). De esta forma, es evidente las discrepancias entre la asignación fenotípica y genotípica de los individuos.

\section{Discusión}

\section{Valoración fenotípica}

La información derivada de visitas a predios y entregada por los productores da idea de constantes situaciones ambientales, sanitarias y de coyuntura económica que modelan día a día las ganaderías en Colombia. En MEZ, la abundante proporción encontrada de razas históricamente asociadas con la producción de leche (p. ej. Holstein y Pardo Suizo) y más recientemente la raza Gyr reconocida por su capacidad para producción de leche, rusticidad y fertilidad a lo largo zonas tropicales y subtropicales $^{(16)}$ se asocian directamente a la búsqueda de animales productivos con adaptación al entorno. 
Como se ha establecido, una proporción superior al $50 \%$ de B. taurus taurus especialmente por las razas lecheras Holstein, Pardo Suizo y Jersey está asociado a mayor producción de leche y una menor respuesta reproductiva en ambientes tropicales, mientras que una proporción mayor de B. taurus indicus en especial de las razas Brahman y Gyr están asociadas a un mayor grado de adaptación e índices reproductivos superiores que los B. taurus taurus, sin dejar de lado los factores netamente relacionados al manejo animal $^{(17,18)}$. Bajo esta premisa, los productores han promovido el mestizaje de los animales, pero sin una orientación de manejo del recurso animal, sin criterios técnicos y en ocasiones sin el conocimiento de la pureza racial de los reproductores ${ }^{(19)}$. Resultado de estas prácticas en este estudio se observó que el desconocimiento total del mestizaje puede llegar hasta un $23.8 \%$ de los animales de sus hatos.

Por su parte, las agrupaciones generadas por APF mostraron cierta simetría entre los grupos PREDTAU, PREDCEB y PREDINTER, solo una ligera desviación fue observada en PREDTAU (+3 \%). Lo anterior deja en evidencia la intención del productor por mantener las proporciones raciales entre los grupos genéticos establecidos por APF, con la intención de explotar mejor el vigor hibrido entre las razas B. taurus taurus y B. taurus indicus más comunes para la producción de leche y a la vez de carne en un sistema tradicional de doble propósito. La aproximación APF, fue un abordaje inicial para la comprensión de los diversos cruces de razas definidas ${ }^{(12)}$, y obedece a protocolos de caracterización racial los cuales aportan información a los análisis genotípicos basados en marcadores moleculares ${ }^{(20)}$.

La amplia gama de cruces y la predominancia del fenotipo B. taurus indicus encontrado en MEZ está bien argumentada en la búsqueda constante por complementar los componentes de mayor producción de leche generalmente otorgada por las razas lecheras del género B. taurus taurus, como Holstein, Pardo Suizo entre otras, y aprovechar la adaptación de los animales $B$. taurus indicus o criollos existentes de los hatos del sistema doble propósito en la Orinoquia ${ }^{(21)}$.

El análisis de componentes principales muestra la alta variabilidad genética de la población y que reduce la correspondencia con las agrupaciones APF propuestas. Esto se traduce en un amplio margen de error cuando se diseñe la estrategia de apareamiento donde se requiere información genotípica o genealógica más precisa de los ancestros que conforman cada individuo y por ende en cada hato ${ }^{(22)}$.

De igual forma, el análisis de componentes principales con razas testigo mostró el alto grado de mestizaje entre $B$. taurus taurus y $B$. taurus indicus. El considerable número de razas testigos usadas en este estudio dan muestra de la compleja relación genética en los hatos doble propósito en la región de estudio y puede ser un indicador de lo que sucede en la gestión genética de los hatos doble propósito en Colombia. Otros estudios presentan esa misma característica, como los reportado para hatos mestizos en África oriental, donde la población estudiada mostro un similar espectro de mestizaje entre Bos taurus taurus y Bos taurus indicus pero sin la presencia de la raza $\mathrm{Gyr}^{(23)}$. 


\section{Designación racial genotípica}

La relativa baja definición de la estructura poblacional obtenida mediante componentes principales para la población MEZ, evidencia de forma clara eventos de mestizaje. Dichos resultados obedecen a un seguimiento genealógico, limitado uso de registros y cruzamientos sin una orientación clara. De hecho, se observa una relación en clina de los individuos en la proyección del PC1 (Figuras 2). Se espera que la frecuencia alélica de los animales MEZ sea intermedia respecto a los animales Bos taurus taurus y Bos taurus indicus $^{(10)}$. Sin embargo, los sistemas productivos tienden a conformar animales cruzados sin control de las proporciones raciales, guiados por la búsqueda de un mayor grado de adaptación a las condiciones ambientales de la región, eficiencia reproductiva y producción de leche ${ }^{(18,19)}$.

Cuando se incluyeron grupos genéticos conocidos en los análisis (Figura 3), se logró proyectar la estructura genética de estas poblaciones y permitió establecer la composición de MEZ, siendo evidente la ubicación en los extremos de los grupos genéticos $B$. taurus taurus, conformada por las razas; HOL, JER, PAR, NOR y BON y el grupo genético $B$. taurus indicus conformado por las razas GYR, BRA, dado que el sistema de producción está enfocado en buscar biotipos animales mejor catalogados para producción de leche y carne. La amplia variabilidad observada en los análisis componentes principales (PC1; Figura 3), coincide con la distribución observada en estudios con hatos mestizos doble propósito en África ${ }^{(23)}$.

La abundante proporción de las razas HOL, GYR y BRA encontradas en la población de estudio mediante los análisis de ADMIXTURE, evidencian el uso de toros reproductores de origen $B$. taurus taurus con mayor afinidad para producción de leche (Holstein) y de origen B. taurus indicus (Brahman y Gyr) con afinidad para la producción de carne y leche ${ }^{(24)}$. No obstante, existen hatos con casos particulares donde el aporte B. taurus taurus puede provenir de otras razas de origen europeo como Pardo Suizo, Normando, Jersey y de razas criollas como la Blanco orejinegro, incluso, pese a su aparente menor tolerancia al trópico cálido, ciertas razas locales pueden suplir las necesidades de hibridación con razas $B$. taurus indicus ${ }^{(25)}$.

Las proporciones raciales predominantes pueden variar por hato, región o país, dependiendo de la disponibilidad de los reproductores (material seminal y animales en pie), orientación y hato base ${ }^{(8)}$ como lo demuestra estudios en hatos mestizos en África oriental, donde la mayor composición de los hatos estuvo dada por las razas Holstein, Friesian (Tanzania y Etiopia) y Ayrshire (Kenia), mientras que la raza Nelore resultó común para los tres países ${ }^{(23)}$.

La diversidad en la gestión de los hatos bovinos doble propósito en Colombia ha sido generalmente asociada a un manejo extensivo, con limitado registro de información productiva de los animales, lo que ha socavado aún más en problemas para establecer biotipos o cruzamientos que brinden mayor heterosis y por ende el mejor desempeño 
productivo $^{(24,26)}$, en este sentido, las aproximaciones genotípicas resultan determinantes para conocer la composición racial y cimentar las bases para la comprensión del desempeño productivo y adaptativo del hato.

\section{Agrupación fenotípica propuesta (AFP) vs Asignación racial genotípica (ARG)}

La agrupación fenotípica propuesta (AFP) ayudó parcialmente a la designación racial del $71 \%$ de los animales en los grupos PREDTAU y PREDCEB, por ende, podría emplearse como una herramienta de orientación en el manejo de cruzamientos o una estrategia para el mejoramiento genético ${ }^{(27)}$. Sin embargo, el $29 \%$ de animales catalogados como PREDINTER resulta compleja su orientación, requiriendo que los animales presenten un fenotipo B. taurus taurus y B. taurus indicus definido.

El rango tan amplio observado en PREDTAU (0.1 a $80 \%)$, PREDCEB (0.1 a 84.2 \%) y PREDINTER (0.1 a $88 \%$ ) para la asignación racial individual hizo visible como la aproximación por ARG favorece la correcta definición de la composición racial, incluso al detectar una considerable proporción para la raza Gyr (65.4 \%) en PREDTAU un grupo donde presume que la proporción de razas B. taurus indicus es mínima o HOL (53.3 \%) en PREDCEB donde se espera una mínima porción de razas B. taurus taurus. Por su parte la porción que presenta mayor dificultad como es PREDINTER reveló que ambos grupos genéticos B. taurus taurus (BON y HOL) y B. taurus indicus (GYR y BRA) alcanzaron valores máximos superiores al $50 \%$. Tal como lo sugieren estudios en animales Brangus y en cruces terminales entre Angus, Charolais y Hereford la aproximación genotípica aportó precisión a la correcta definición de la composición máxima para la raza Angus ${ }^{(22)}$ y los cruces terminales ${ }^{(28)}$.

La consideración de una clasificación APF requiere que los animales presenten los rasgos fenotípicos de las razas, o que contemplen una estructura fenotípica con rasgos combinados. Sin embargo, aunque los individuos presenten un fenotipo similar, es posible encontrar diferencias alélicas asociadas a otras características de interés, como rendimiento en canal, producción de leche, calidad de la carne y la leche ${ }^{(29)}$. Una amplia divergencia entre métodos APF y ARG fue observada cuando estas fueron comparadas entre sí, un amplio y variado rango de aporte genético (determinado por ARG) de diversas razas fue encontrado $(0.1 \%$ hasta el $88.9 \%)$, lo cual deja al descubierto las erróneas interpretaciones a las que ha estado sumergido el manejo de un hato cuando éste se ciñe a la perspectiva de su apariencia, contrario a lo que ocurre en hibridaciones orientadas por información genealógica, como es el caso de animales para la producción de carne, buscando el equilibrio en las composiciones genéticas y explotar mejor el vigor hibrido $^{(22)}$. Así mismo, se sabe que no todos los cambios fenotípicos pueden ser atribuidos a cambios genéticos. Algunas diferencias en el color del pelo pueden ser atribuido a factores no genéticos como la edad, intensidad de la radiación solar o por la combinación 
de los factores genéticos y no genéticos ${ }^{(28)}$. Esta es la razón por la cual es posible observar diferencias en la correspondencia fenotípica y genotípica, y es aquí donde las evaluaciones genotípicas cobran gran valor por su contribución en la precisión de las determinaciones y una herramienta adicional para la orientación de los esquemas de apareamiento más acordes a la realidad ${ }^{(22,25)}$ y que contribuyan a mejorar los indicadores productivos al recortar tiempo para alcanzar los objetivos del productor, al permitirle a éste de forma temprana identificar y seleccionar animales cuya composición racial diverja de las metas establecidas, en otras palabras, haciendo del sistema productivo más rentable ${ }^{(22)}$. La definición de las razas que más aportan a la composición del mestizaje en cada hato da idea de la orientación que ha recibido, por ende, es el indicativo fiel de los indicadores productivos en un hato cuyo enfoque incluye la producción de leche y carne $^{(23)}$.

Previos reportes sugieren que las predicciones genotípicas han permitido corregir eficazmente erróneas asignaciones basadas en información genealógica de bovinos $\operatorname{cruzados}^{(9)}$. Por otro lado, la asignación racial genotípica incorpora elementos de la demografía de las poblaciones y permite definir estrategias de manejo y conservación tanto en términos de población ${ }^{(30)}$, como en el manejo de las frecuencias alélicas para genes de interés productivo asociados al crecimiento, calidad de la canal, calidad de la leche, la reproducción y la adaptación al trópico ${ }^{(9,25)}$.

\section{Conclusiones e implicaciones}

El conocimiento de los productores y la aproximación APF (PREDTAU, PREDCEB y PREDINTER) aportaron a dilucidar parcialmente la composición racial de un hato mestizo de bovinos doble propósito y sobre el manejo genético que un hato lleva en búsqueda de un equilibrio entre los grupos genéticos $B$. taurus taurus y $B$. taurus indicus. Sin embargo, se observó un amplio rango de error bajo esta metodología, por lo que una porción del hato podría asignarse de forma errada en un grupo determinado y desencadenar el ya conocido mestizaje sin orientación. La aproximación ARG permitió de forma efectiva identificar 7 grupos genéticos en la conformación de los hatos MEZ, con ello permitió de forma clara robustecer los métodos convencionales basados en apreciaciones fenotípicas como APF para definir la composición racial de bovinos mestizos doble propósito. La ARG tiene la capacidad de garantizar una amplia precisión en las predicciones de composición racial individual y del hato, con lo cual podría aportar de forma segura y rentable al desarrollo de estrategias de apareamientos dirigidos o de cruzamiento que garanticen mejor aprovechamiento del vigor hibrido con un equilibrio entre la producción de leche, carne y contemple su adaptación reconociendo las condiciones tropicales donde se desarrolla este sistema de producción como es el piedemonte del departamento de Meta. 


\section{Agradecimientos y conflicto de intereses}

Los autores agradecen a la Corporación Colombiana de Investigación AgropecuariaAGROSAVIA adscrita al Ministerio de Agricultura de Desarrollo Rural de ColombiaMADR por la financiación de esta investigación. A los productores de ganado doble propósito que hicieron parte del proyecto y al laboratorio de genética molecular del C.I. Tibaitatá por los análisis genómicos. Este trabajo fue parte del proyecto "Estrategias integrales y participativas de fortalecimiento tecnológico del sistema bovino doble propósito del piedemonte llanero (Fase 1)".

\section{Literatura citada:}

1. Federación Colombiana de Ganaderos- FEDEGAN. Cifras de referencia del sector ganadero colombiano.2018. https://www.fedegan.org.co/estadisticas/documentosde-estadistica.

2. Ortega LE, Ward RW, Andrew CO. Technical efficiency of the dual-purpose cattle system in Venezuela. J Agr Appl Economy 2007;39:719-733.

3. Pariacote FA. Perspectivas de mejoramiento genético del bovino criollo. En Duran C, Campos R, editores. Perspectivas de conservación: Mejoramiento y utilización de recursos genéticos criollos y colombianos en los nuevos escenarios del mejoramiento animal. Palmira-Valle del Cauca. UN-Palmira. 2008:17-30.

4. Vergara GOD, Flórez MJM, Hernández PMJ, Yaguna GCJ, Manco JC, Barrios RTE, Rico CJ. Efectos raciales, de heterosis y parámetros genéticos para peso al nacer en una población multirracial de ganado de carne en Colombia. Livest Res Rural Develop 2014;26(58).

5. Echeverry ZJ, Salazar RVE, Múnera D. El cruzamiento como estrategia para mejorar la rentabilidad de hatos lecheros. Revista Lasallista de Investigación. 2006, 3 (juliodiciembre):<http://www.redalyc.org/articulo.oa?id=69530209> ISSN 1794-4449. Consultado 25 Abr, 2019.

6. Elzo MA. Evaluación Multirracial de Bovinos en Colombia: desde la genética a la genómica. Departamento de Ciencias Animales, Universidad de Florida, Gainesville, FL. Estados Unidos. 2011.

7. Frankham R, Ballou JD, Briscoe DA. Introduction to conservation genetics. Cambridge, UK: Cambridge University Press; 2002.

8. Gorbach DM, Makgahlela ML, Reecy JM, Kemp SJ, Baltenweck I, Ouma R, et al. Use of SNP genotyping to determine pedigree and breed composition of dairy cattle in Kenya. J Anim Breed Genet 2010;127:348-351. 
9. Akanno EC, Chen L, Abo-Ismail MK, Crowley JJ, Wang Z, Li C, Basarab J, et al. Genomic prediction of breed composition and heterosis effects in Angus, Charolais and Hereford crosses using 50K genotypes. Can J Anim Sci 2017;97:431-438.

10. Bolormaa S, Hayes BJ, Hawken RJ, Zhang Y, Reverter A, Goddard ME. Detection of chromosome segments of zebu and taurine origin and their effect on beef production and growth. J Anim Sci 2011;89(7):2050-60.

11.Otero AE, Mosquera AL, Silva CG, Guzmán VJC. El Piedemonte. La Orinoquia de Colombia. Bogotá: Banco de Occidente 2014. ISBN 95896774968.

12.Villasmil IW, Aranguren-Méndez JA. Clasificación fenotípica en vacas mestizas. En: González-Stagnaro C, Soto-Belloso E, editores. Manual de Ganadería Doble Propósito. Maracaibo-Venezuela: Ediciones Astro Data, S.A; 2005;8(1):283-290.

13. Li Y, Levran O, Kim J, Zhang T, Chen X, Suo Ch. Extreme sampling design in genetic association mapping of quantitative trait loci using balanced and unbalanced casecontrol samples. Sci Rep 2019;9:15504. https://doi.org/10.1038/s41598-019-51790W.

14. R Core Team R: A language and environment for statistical computing. R Foundation for Statistical Computing 2014: Vienna, Austria. http://www.R-project.org/.

15. Alexander DH, Novembre J, Lange K. Fast model-based estimation of ancestry in unrelated individuals. Genome Res 2009;19(9):1655-1664.

16. Neves HHR, Carvalheiro R, Fries LA, Queiroz SA. Uso combinado de sêmen sexado e acasalamento dirigido sobre uma população de bovinos de corte submetida a seleção: estudo de simulação. Rev Brasileira Zoot 2009;38:2368-2374.

17. Perdomo-Carrillo D, Perea F, Soto-Belloso E, Piña-Monsalve J, Moratinos-López P, Martínez-López M, Perea-Brugal M. Efecto de la raza paterna sobre el desempeño productivo de un rebaño de vacas mestizas doble propósito en Venezuela. Maskana 2017;8:141-143.

https://publicaciones.ucuenca.edu.ec/ojs/index.php/maskana/article/view/1509

18. Zambrano R, Santos H, Contreras R, Moreno A, Chirinos Z. Características productivas de un rebaño mestizo bovino doble propósito comercial en Venezuela. Actas Iberoam Conserv Anim 2013;3:15-19.

19. Galeano AP, Manrique C. Estimación de parámetros genéticos para características productivas y reproductivas en los sistemas doble propósito del trópico bajo colombiano. Rev Facultad Med Vet Zoot 2010;57(II):119-131.

20. FAO. Food and Agriculture Organization of the United Nations. Phenotypic characterization of animal genetic resources. FAO Animal Production and Health Guidelines. Rome. 2012. 
21.Salamanca-Carreño A, Vélez TM, Bentez MJ. Efectos no genéticos sobre la reproducción en vacas mestizas con predominio Bos indicus en el municipio de Arauca, Colombia. Rev Invest Vet Perú 2017;28(1):101-109. https://doi.org/10.15381/rivep.v28i1.11749.

22. Wang Y, Wu X-L, Li Z, Bao Z, Tait RG Jr, Bauck S, Rosa GJM. Estimation of genomic breed composition for purebred and crossbred animals using sparsely regularized admixture models. Front Genet 2020;11:576.

23. Strucken EM, Al-Mamun HA, Esquivelzeta-Rabell C, Gondro C, Mwai OA, Gibson JP. Genetic tests for estimating dairy breed proportion and parentage assignment in East African crossbred cattle. Genet Sel Evol 2017;49(1):67. doi.org/10.1186/s12711-017-0342-1.

24. Cortés H, Aguilar C, Vera R. Sistemas bovinos doble propósito en el trópico bajo de Colombia. Modelo de simulación. Arch Zootec 2003;52:25-34.

25. Ahmad SF, Panigrahi M, Chhotaray S, Pal D, Parida S, Bhushan B, Gaur GK, et al. Revelation of genomic breed composition in a crossbred cattle of India with the help of Bovine 50K BeadChip. Genomics. 2020; 112(2):1531-1535. doi: 10.1016/j.ygeno.2019.08.025. Epub 2019 Aug 28. PMID: 31472242.

26. Ramírez-Toro EJ, Ocampo-Gallego RJ, Burgos-Paz WO, Elzo MA, MartínezSarmiento RA, Cerón-Muñoz MF. Estimación poligénica y genómico-poligénica para características de crecimiento en ganado Blanco Orejinegro (BON). Livest Res Rural Develop 2019;31:30.

27. Aranguren-Méndez J, Román R, Villasmil-Ontiveros Y. Evaluación genética de la ganadería mestiza doble propósito en Venezuela. XX Reunión ALPA, XXX Reunión APPA-Cusco-Perú. Arch Latinoam Prod Anim 2007:15(Supl 1):241-250.

28. Akanno EC, Chen L, Abo-Ismail MK, Crowley JJ, Wang Z, et al. Genomic prediction of breed composition and heterosis effects in Angus, Charolais, and Hereford crosses using 50K genotypes, Can J Anim Sci 2017;97(3):431-438.

29. Orgogozo V, Morizot B, Martin A. The differential view of genotype-phenotype relationships. Front Genet 2015;6:179.

30. Gobena M, Elzo MA, Mateescu RG. Population structure and genomic breed composition in an Angus-Brahman crossbred cattle population. Front Genet 2018; 9:90. https://doi.org/10.3389/fgene.2018.00090. 